\title{
Case study of a successful learner's epistemological framings of quantum mechanics
}

\author{
Vesal Dini* \\ Department of Physics \& Astronomy, Tufts University, Medford, Massachusetts 02155, USA \\ David Hammer \\ Departments of Education, and Physics \& Astronomy, Tufts University, \\ Medford, Massachusetts 02155, USA \\ (Received 10 August 2016; published 15 May 2017)
}

\begin{abstract}
Research on student epistemologies in introductory courses has highlighted the importance of understanding physics as "a refinement of everyday thinking" [A. Einstein, J. Franklin Inst. 221, 349 (1936)]. That view is difficult to sustain in quantum mechanics, for students as for physicists. How might students manage the transition? In this article, we present a case study of a graduate student's approaches and reflections on learning over two semesters of quantum mechanics, based on a series of nine interviews. We recount his explicit grappling with the shift in epistemology from classical to quantum, and we argue that his success in learning largely involved his framing mathematics as expressing physical meaning. At the same time, we show he was not entirely stable in these framings, shifting away from them in particular during his study of scattering. The case speaks to literature on students' epistemologies, with respect to the roles of everyday thinking and mathematics. We discuss what this case suggests for further research, with possible implications for instruction.
\end{abstract}

DOI: 10.1103/PhysRevPhysEducRes.13.010124

\section{INTRODUCTION}

Consider a group of students working on the problem of a particle incident upon a rectangular potential barrier. They write the time-independent Schrödinger equation, and they solve it correctly, using a worked solution as a guide. Do they consider the physical meaning of the calculations, such as to recognize how a particle with energy greater than the barrier might reflect, or that one with energy less than the barrier might pass through? Or do they write down the functions and move on to the next problem?

We are interested in how students frame their work in learning quantum mechanics (QM), in particular with respect to the roles of everyday experience and of mathematics. These are aspects of student epistemologies, which have had significant attention in research on learning in introductory courses but less in physics education research (PER) with respect to QM.

Most research on learning and teaching in QM has focused on conceptual understanding. Researchers have studied student learning about probability amplitudes, for an example relevant to the students above, including specifically in the case of tunneling [1-3]. There have been studies on single dimension particle dynamics [4], time dependence [5,6], and a range of other concepts, including the uncertainty principle

\footnotetext{
*vesal.dini@tufts.edu

Published by the American Physical Society under the terms of the Creative Commons Attribution 3.0 License. Further distribution of this work must maintain attribution to the author $(s)$ and the published article's title, journal citation, and DOI.
}

[7], wave particle duality [7-10], quantum measurement [11-14], operators [15], the Stern-Gerlach experiment [16], Hilbert space [17], and Dirac notation [18].

Studies of students' conceptual understanding have influenced and taken place in contexts of instructional reforms. Researchers have redesigned courses to increase student engagement through classroom group activities [19], peer instruction [20], and interactive lectures [21]. They have also re-envisioned entire course content, such as to center on the Stern-Gerlach experiment and spin dynamics $[22,23]$, or on the major conceptual and interpretive questions of QM $[24,25]$.

Researchers have also devised instructional units on specific topics, such as conduction models [26], quantum effects in LEDs and luminescent devices $[27,28]$, probability and probability density $[29,30]$, models of the atom [31], potential energy diagrams [32], and the photoelectric effect [33-37]. A great deal of work has leveraged technology to help students build intuitions about QM concepts, such as in Quantum Interactive Learning Tutorials [38,39], Physics Applets [40,41], and the Visual QM project [42-46].

Along the way, too, researchers have developed and refined instruments to assess students' understanding, multiple choice, or short-answer tests at the graduate and undergraduate levels [47-54]. These have served as tools for research on learning and instructional development in QM much as the Force-Motion Concept Evaluation [55] and the Force Concept Inventory [56] did for Newtonian mechanics.

There has thus been a great deal of research on learning in QM, but there has been relatively little attention at the level of how students approach learning. 


\section{A. The relevance of student epistemologies}

Returning to the group solving the rectangular potential barrier, there is more to consider than their conceptual understanding of probability amplitudes, reflection, and transmission. There is also how they approach the problem and learn from it, how they draw on worked solutions as templates, whether and how they consider the physical situation the equations depict.

Studying learning in introductory physics, Hammer [57] described a student who treated equations as meaningful expressions of ideas. "Tony" did that both in solving problems, such as referring to an algebraic expression as having "said" where a force would be acting, and in reflecting on his experience in the course, much of which he saw as "a matter of putting common sense into equations." Challenged to account for ideas beyond common sense, he explained, "I usually tend to modify my common sense during the year." Other students were more like "Roger," who spoke of equations as coming from the textbook and showed little concern that calculations fit with his physical intuition. Like Tony, Roger's views were evident both in his behavior and explicit comments, such as in explaining he would "just look at the book" to learn or to teach the formula $v=v_{0}+a t$.

Research on learning in QM has moved relatively quickly to study epistemologies [58-61], perhaps in part because matters of epistemology have been and remain salient in QM among physicists [62,63]. Many courses include references to debates between Bohr and Einstein, and between Heisenberg and Schrödinger, over what sort of knowledge science can and should provide, including specifically with respect to the role of mathematics and its relationship to "everyday thinking." Most at some point present the epistemology of the Copenhagen interpretation.

In these ways, QM raises questions about what kind of understanding to expect, of what "sense making" entails, including and, in particular, with respect to the role of mathematics. A stance like Tony's, of learning physics as "putting common sense into equations" and "modify[ing] common sense" as the formalism demands, seems productive in classical mechanics, in line with Einstein's "The whole of science is nothing more than a refinement of everyday thinking" [64]. In QM, that stance has needed refinement. For physicists as for students, conceptual understanding in QM ultimately anchors in mathematics, and learning QM requires an epistemological view of mathematics as "saying things" with physical meaning.

\section{B. Studying the epistemology of a successful student}

This article is part of a larger study of student epistemologies in learning QM [65]. Here, we focus on a particular, successful graduate student we call "Bailey," whom the first author followed for a year of study, in first and second semester QM. Bailey mostly showed a stance of looking for meaning in the mathematics, both in explicit comments and in how he approached problems. He seemed to shift from that stance, however, in moments of problem solving during the second semester course, treating mathematics as distinct from sense making. We show evidence for these different epistemologies, discuss how they may have arisen and become stable in their respective contexts, and consider their relevance for Bailey's learning.

The remainder of this paper is divided into four main sections. Section II provides background on PER around student epistemologies, both at the introductory level and in QM. Section III presents the case study, including methods and the two main episodes that indicate different epistemologies. Section IV provides an explanation for why these differences may have arisen, and Sec. V discusses implications of this work.

\section{RESEARCH ON STUDENT EPISTEMOLOGIES}

At least as early as 1985 [66,67], PER has considered the nature and roles of epistemologies in introductory physics. Earlier work took place mostly in qualitative studies, through interviews, student journals and written surveys, and classroom observations [57,68-72]. These supported researchers' developing instruments for quantitative study $[73,74]$.

Across this work, the evidence shows students come out of most courses more inclined to think of knowledge in physics as piecemeal facts and formulas provided by authority and essentially separate from their experience of the physical world. By the same token, they are more inclined to approach learning as a matter of memorizing information and rehearsing solution methods. These results have motivated curriculum design and pedagogical strategies to promote more productive views $[68,75,76]$.

Broadly speaking, these curricula work toward students coming to see knowledge in physics as a principled system of understanding, building from and coherent with appropriately refined everyday thinking. As Etkina et al. [75] put it, students should "become familiar with the epistemology of the scientific community." And they should approach learning as sense making, a pursuit of understanding that reflects the practices of the discipline. A number of course reforms have shown evidence of success, in gains on the Colorado Learning Attitudes About Science Survey (CLASS) and Maryland Physics Expectations Survey (MPEX) surveys [77].

New questions arise for research on student epistemologies in QM, in particular over the role of mathematics and the relationship between everyday thinking and the principled system of knowledge in physics. Before we turn to that work, we review a relevant matter of theoretical development in models of students' epistemologies.

\section{A. Fixed and dynamic models of epistemologies}

Early research on students' epistemologies modeled them mainly as Piagetian stages of development $[78,79]$. 
Schommer [80] introduced the possibility of multiple dimensions of development; Hofer and Pintrich [81] argued for a view of epistemologies as intuitive theories. These accounts described epistemologies as individuals' stages of development or stable theories, possibly with multiple aspects, but in essentially unitary forms.

Roth and Roychoudhury [72] was an early exception, arguing that "it might be more appropriate to speak of epistemological positions only in specific contexts rather than as descriptors of an individual's views in general." Subsequent studies have produced extensive evidence of contextual variability and dynamics, from students' responses to survey data $[69,82]$ as well as their reasoning in situ [83-85]; Bromme's and colleagues' [86,87] experiments have manipulated contexts to cue different epistemologies.

Hammer and Elby [88,89] proposed a model of "epistemological resources" as an alternative ontology to models of stages or theories. On this view, people have a myriad of ways of thinking about knowledge, for example, as a kind of "stuff" one gets from a source, or can create for oneself. People have resources for understanding knowledge in a wide variety of forms, such as rules or facts or nonverbal awarenesses, as well as for understanding related activities, such as guessing or supposing or deriving. And any of us invokes different sets of these resources in different situations.

Redish [90] connected that perspective to literature on frames and framing [91-93], proposing "epistemological framing." A frame is a "structure of expectations" about what is taking place [93], the tacit answer to the question "What is it that's going on here?" [92]. It may involve expectations with respect to many aspects of the situation, including social (e.g., "how and with whom am I interacting?"), affective ("how am I feeling about this?"), positional [94] ("what is my role here?"), among others.

Epistemological framing, then, refers to learners' forming a sense of "what is it that's going on here with respect to knowledge?" That could include a sense of what kind of knowledge is relevant (e.g., common sense or formulas or both) or the kind of activity (e.g., guessing, inventing, deducing); in this way forming a sense of what is going on with respect to knowledge involves sets of epistemological resources, activated in concert.

A number have developed dynamic accounts of epistemologies in upper division courses [61,95]. Work by Tuminaro and Redish [96] is specifically relevant below, in their account of "epistemic games" involved in studying physics. Two of these will be especially relevant below, concerning the relationship between mathematics and conceptual understanding. Mapping meaning to mathematics begins "from a conceptual understanding of a physical situation" and uses it as the basis for constructing mathematical expressions. Mapping mathematics to meaning is the opposite: "students begin with a physics equation" and use it as the basis for constructing a conceptual understanding.

\section{B. Research on student epistemologies in QM}

Bing and Redish [59] built from the notion of framing to analyze students' problem solving in QM, specifically with respect to mathematics. They identified framings, primarily evident in how students warranted claims: (i) calculation, or algorithmically following a set of established computational steps; (ii) physical mapping, between a mathematical symbolic representation and features of a physical system; (iii) invoking authority, finding information from a reliable source such as a textbook, and (iv) mathematical consistency, making use of the regularity and reliability and consistency of mathematics across different situations.

They showed these four framings emerging and disappearing dynamically in student discourse, on local time scales. They also show how these framings can nest within larger time scales, one subject, for example, "nesting his computation within a larger scheme of supporting his physical mapping argument." Part of expertise, they argue, is the ability to initiate and coordinate framings in this way, much like Schoenfeld's [97] earlier arguments regarding expertise in mathematical problem solving.

Baily and Finkelstein found students' epistemologies in QM vary with context and can be influenced by instruction. For example, they compared subjects' responses across questions [58]. One presented fictional students' explanations of double-slit electron interference, reflecting realist or quantum interpretations. ${ }^{1}$ Subjects wrote brief essays agreeing or disagreeing with each explanation. Another asked subjects to agree or disagree that "an electron in an atom has a definite but unknown position at each moment in time." Most subjects (61\%) were inconsistent across questions, varying between quantum and realist perspectives.

In another study, Baily and Finkelstein compared students' thinking after hearing contrasting explanations [98]. In one, the instructor described an electron in a double-slit experiment as passing through both slits and interfering with itself before being detected on the screen. In the other, the instructor asserted that one cannot characterize the trajectory of an electron, since there is no way of determining its actual path without destroying the interference pattern. Students from the first class were more likely to show quantum mechanical reasoning; students from the second to take a realist perspective.

Baily and Finkelstein [24] drew on these findings in designing their reformed course in which "students developed more consistent interpretations of quantum phenomena, more sophisticated views of uncertainty, and greater interest in quantum physics" (p. 2) than in conventional courses. Most relevant here, Baily's and Finkelstein's reforms emphasize drawing physical meaning from

\footnotetext{
${ }^{1}$ Much of Baily's and Finkelstein's (2014) analysis focuses on the courses'and students' ontologies (e.g., what kind of thing is an electron), which connects to epistemologies (what kind of knowledge is QM).
} 
mathematical expressions and calculations, on the view that students learning classical or QM may apply equations without meaningful understanding.

Levrini and Fantini [99] examined data from an experimental course they taught for Italian students in upper secondary school, grade 13. They examined students' discussions concerning the role of the formalism, in students arguing, for example, that the "formalism was necessary but not sufficient to have the feeling of understanding: comprehension requires the 'formal mechanism' to be interpreted also in terms of (smooth) links to ordinary language and classical description" (p. 1903). They drew on these data to support their arguments that instruction in QM should compare and contrast epistemologies in classical and QM, and to show that students were able to engage productively with the ideas.

Other studies of learning in QM bear on students' epistemologies and epistemological framing. Mason and Singh [100] found most students in their QM course did not learn from mistakes on exam problems. They recount data from interviews, such as one student who explained his poor performance on an exam problem as "not remembering how to manipulate with bra and ket. Basically that's all it is." Another more successful student, in contrast, saw mistakes as signaling gaps in his understanding.

Brookes and Etkina [101] analyzed the use of metaphors in QM (e.g., a "potential well") and hypothesized that a student could be "distracted by an overly literal interpretation." Gire and Price [102] showed evidence of student reasoning interacting with features of Dirac, wave function, and matrix notations. Modir and Sayer [61] examined students' group problem solving on a free particle problem and identify a mathematical resource called "pulling out" that aids them in sense making. In the terms we introduced above, the three studies are examples of contextual dynamics in resources and framing, as different language or representations cue different patterns of reasoning.

\section{From classical to quantum}

In many respects, research to date on student epistemologies in QM is continuous with prior work and leads to similar conclusions: Students must learn to see physics as a coherent body of knowledge, themselves as agents in seeking that coherence, and mathematics as expressing and supporting meaningful understanding. They have resources for understanding physics in these ways; part of progress toward expertise involves developing awareness of and stability in productive epistemologies. Bing and Redish's arguments, for example, would apply to upper division courses in classical mechanics.

In other respects, as Baily and Finkelstein and Levrini and Fantini argued, QM raises new issues of epistemology, for students as in the history of physics. It is difficult to see QM as "a matter of putting common sense into equations," in student Tony's words [57], or as a refinement of everyday thinking, in Einstein's [64]. Consider the central and constructive role of everyday thinking in classical thought experiments [103,104]. In his dialogues, Galileo routinely built on what was obvious to "Simplicio," the voice of common sense, such as regarding the steady motion of a ship on a calm sea to argue that objects move without slowing in the absence of external influence. Research on student epistemologies in introductory mechanics has long associated success with a view of physics as related to-and consistent with-everyday experience. Apparently "counterintuitive" results are explained by reference to other aspects of intuition; successful students work to reconcile the contradiction. Mathematics also plays a central and essential role, certainly, but for Einstein as for successful students in introductory courses, it remains deeply connected to and continuous with everyday thinking.

There are also thought experiments in QM, of course, but they do not appeal in the same way to everyday experience. Rather, they pursue and explore conceptual implications and connections with mathematical principles of QM. Schrödinger's cat, for example, explores the result of QM thinking leading to an intuitively problematic result. Einstein's, Podolsky's, and Rosen's famous thought experiment of a pair of systems shows how QM premises lead to what the authors argued was a contradictory result. To be sure, these and other famous thought experiments (Heisenberg's microscope, Wheeler's delayed choice, Wigner's friend) all took place as part of professional epistemological debates over what kind of knowledge QM provides.

For physicists or students who have been studying classical physics as a "efinement of everyday thinking, QM requires not only new conceptual material, but also a change of epistemology. It is a shift with respect to the role of everyday thinking and experience and, at the same time, with respect to the role of mathematics. Historians of physics have examined what happened for the field. What, we are interested to understand, happens for students?

Prior research in introductory courses, including the second author's [57], has argued for instruction that cultivates students' understanding physics as a refinement of everyday thinking. If that is the favorable epistemology in classical physics, what happens for students as they progress in to QM? Or, if that epistemology needs to change as students proceed to QM, perhaps it is not one to cultivate in the first place. Our purpose in this paper is to begin to address these questions.

In sum, we hope this article will contribute to the literature in three ways. First, as we have shown, there has been relatively little research so far focusing on student 
epistemologies in QM. Given the salience of epistemology in QM for physicists, it seems an area for further attention. Second, more specifically, it will begin to address the questions in the previous paragraph, to consider whether and how students experience and manage a shift in epistemology in studying QM. Finally, this article is part of a larger program of research to study students' epistemological development over semesters and years [105].

We report on our analysis of one student's work in graduate QM. We have chosen Bailey, as we call him, for this article because he was especially successful in learning QM, both by the professors' assessments and ours, and because he was especially articulate during interviews. As we show, his interviews were rich in evidence regarding his grappling with a shift of epistemology.

\section{CASE STUDY: A SUCCESSFUL STUDENT'S EPISTEMOLOGIES}

Bailey was one of eight graduate students who volunteered to participate. There were also five undergraduates. Dini [65] presents the work of the project in full.

\section{A. Methodology}

Given our expectation, discussed above, that epistemologies are context dependent, we tried to design a study that would stay close to the contexts of learning subjects experience as students.

One approach would be to collect data simply by observing and recording students' work. ${ }^{2}$ These courses, however, did not afford sufficient evidence in themselves: students speak relatively little during lectures, and it was logistically not possible to record student study sessions. Therefore, we chose to conduct interviews closely connected to students experiences in the courses. Research subjects recounted what they were doing, discussed points of interest or difficulty, and worked through homework or exam problems. The interviews had an open structure, which allowed the researcher, the first author, to find and pursue matters of interest as they arose, as the data below will illustrate.

This approach also allowed us to consider how students' were framing the interview activity itself, whether, for example, as an examination of their understanding, as an opportunity to prove their intelligence, or, as we hoped, as an open inquiry [107]. This is one advantage of an interview over, for example, a questionnaire; another is that the interviewer has the chance to respond to subjects' framings in the moment.

The first author, Vesal, interviewed subjects every 3 to 4 weeks, during the semesters. He started each interview with an open question, "How are things going in quantum?" and

\footnotetext{
${ }^{2}$ Shubert and Meredith [106] recently described stimulated recall interviews, which we see as a promising method.
}

from there asking the subjects to elaborate on issues they raise. We took this approach to investigate what subjects were concerned with, helping them frame the interviews as a venue for their sense making [107]. In moments when subjects did not have more to say, he would also ask some more focused questions, for the subjects to talk about particular aspects of the course including lectures, homework sets, and examinations, as well as about specific content. Regardless of the particular task, he tried to keep comments limited to clarifications or restatements of students' ideas or explanations.

The resulting interviews provided explicit and tacit evidence of epistemology. We gathered evidence from subjects' statements about QM, how the courses were taught, or their approaches to learning. We also looked for evidence in their behavior, including their selfdescriptions of how they studied for exams, solved problems, or used lectures.

Our goal for the analysis was to first focus on what was happening in the interviews without employing an overly narrow analytical lens and looking for a specific set of epistemologies that might arise in particular situations. Rather, we were interested in recognizing epistemologies that emerged from the data. This is representative of a grounded theory approach [108].

We searched for evidence of epistemology through markers in discourse [57,59,93,107,109]. Researchers have long explored ways of using discourse to make claims about students' epistemologies, and we built on this prior research. Our process involved writing up a detailed account of each interview while identifying epistemologies both in short instances and longer episodes within them. Examples of the kinds of markers we found, and the categories of epistemology they correspond to, are presented in Table I.

TABLE I. Examples of evidence.

\begin{tabular}{ll}
\hline \hline Epistemology & \multicolumn{1}{c}{ Discourse marker } \\
\hline References to knowing & "We construct the ideas..." \\
& "I don't know what my \\
confusion is..." & "I want to understand..." \\
"I need to remember this idea..." & "The professor said so..." \\
Aims of knowledge & "I built the idea from \\
mathematics..." & "I used my intuition..." \\
Sources of knowledge & "The experiment proved it..." \\
"I need to know a fact before \\
moving on..." \\
"We can only know to a certain \\
probability..." \\
"None of these ideas is related..."
\end{tabular}


Vesal interviewed Bailey for $1 \mathrm{~h}$ nine times over the year, five in the first semester and four in the second. As we noted, Bailey was both a successful of QM and articulate in these interviews, rich in evidence of epistemology. Below, we present four such examples of evidence. For the first two examples, we perform a close analysis that makes reference to categories presented in Table I. For the last two examples, we provide shorter summaries. Our goal in this is to help the reader get a feeling for how we went about making claims from Bailey's discourse.

Example 1: Bailey explains his sense of the role of mathematics in studying the topic of angular momentum.

1 Bailey: We take time to sort of build up this,

2 um, you know this sense of how we're

3 going to operate mathematically and talk

4 about these things mathematically before

5 we're then able to actually look at any

6 sort of physical representations that

7 are, um, are either consequences of that

8 or in which that sort of math framework

9 that we've built up is helpful to us, um,

10 and so we've just started to get to sort

11 of you know why do we care about this

12 stuff, why have we built up this

13 mathematical framework, you know like, oh

$14 \mathrm{OK}$, these are the physical situations in

15 which it comes into play.

Bailey describes mathematical knowledge as being constructed (lines 1-3, lines 8-9), a form of "Reference to knowing" listed in Table I. After becoming sufficiently familiar with the mathematical framework, he suggests that physical systems can be understood through it (lines 5-9). In this, Bailey implicitly communicates an "Aim of knowledge": to understand the physical world. He also offers that physical representations could be a consequence of how the mathematics works out (lines 6-7), an indication that it acts as a useful "source of knowledge." In sum, we took this excerpt as evidence of Bailey's professing a coherence with mathematics ("structure of knowledge"), because it describes the mathematics as integrated with and supportive of ("helpful to us") understanding the physics.

Example 2: At the end of a round of sense making about a problem involving the coupling of angular momentum of two spin- $1 / 2$ particles, Bailey arrives at an explanation for why a state $|J=2, M=0\rangle$ in the coupled representation corresponds to three states with unequal spread in an uncoupled representation.

1 Bailey: It starts to make more sense, that sort

2 of probabilistic analogy that I wanted to

3 get to before but couldn't really find a

4 way to explain it, um, you know, just

5 sort of looking at the, the ways that we

6 can get to these various states, it makes

7 sense that it wouldn't be a third, a
8 third, a third, right, because if we

9 start from the very top of our ladder

$10[\ldots]$ and then if we just sort of cascade

11 down through the possibilities [...] we

12 see that there are more pathways to get

13 to that 0,0 state as opposed to the $1,-1$

14 or the $-1,1$ state.

Bailey's "Aim of Knowledge" in this moment is to generate a sensible explanation (lines 1-4). He arrives at one, supported by a tacit view of mathematics as conveying physical meaning (source of knowledge): there are more ways to get to the 0,0 state than to the others, making it more likely to measure (lines 8-14). Bailey expects and finds coherence in his thinking around the idea of angular momentum states, their representations, and the probability of measuring them in the unfolding mathematics of the problem that encodes meaning to be parsed (structure of knowledge).

Example 3: While exploring the evolution of atomic models, Bailey begins to sense make about the notion of size with respect to the nucleon:

1 Bailey: So I wonder then when we talk about the

2 size of the nucleus of something, what is

3 the connection between the size of that

4 nucleus and the sizes of a proton or

5 neutron that we say are making it up, and

6 that probably is a much more subtle thing

7 than it seems [...] the nuclear size

8 might not be something that's a physical

9 size, it might be sort of an effective

10 size that's sort of dictated by some

11 property of it [...] when we're looking

12 at something that's that fine, that's

13 that small and quantum mechanical, it's

14 not it seems to me particularly

15 meaningful to talk about its like

16 physical size [...] It's like its

17 interaction size, it's like its sphere of

18 influence that sort of matters more cause

19 we never really have particles like

20 interacting with one another by making

21 physical contact.

We took this as evidence of Bailey seeking coherence in his intuitive knowledge while trying to align the idea of an object's "size" with a distance at which it interacts (a more principled understanding of size than the common sense "How big is it?").

Example 4: In between discussing concepts in scattering,

Bailey describes the development of his epistemology.

1 Bailey: We've talked about how logic sort of

2 goes out the window, but I don't think it

3 does anymore. I think that it's just that

4 it's a different logic that we need to

5 build, right, [...] it's a new realm and

6 a new sort of way of thinking that needs 
7 to be developed, but you know with that

8 new realm or with that new way of

9 thinking there comes a, a logic that is

10 still, you know, reasonable. [...] It's

11 not like things that happen in this

12 [quantum] world are then unpredictable

13 and, and crazy, they work perfectly

14 within that paradigm, and that's why we

15 build it.

We took this as evidence of Bailey's awareness of constructing his epistemology, as he contrasts his earlier view that intuition is useless, with a later view that, to the extent he has built it, quantum intuition is consistent and useful.

\section{B. Results: Evidence of Bailey's epistemological framings}

Here we present detailed analyses of two particular episodes over the year, concerning Bailey's approach and efforts to understand the Aharanov-Bohm (AB) effect, in the first semester, and scattering theory in the second. Both show what we characterize as Bailey's stable, articulate commitment to constructing a conceptualization of QM connected with the mathematical formalism. The first reflects a pattern evident throughout Bailey's interviews of his framing mathematics as an expression of conceptual meaning. The latter showed a break in that pattern. We first present each episode with our analysis, followed by our account of the different dynamics.

\section{Studying the AB effect: Mathematics as an expression of conceptual meaning}

Shortly before the second interview of the first semester, the class had been studying the $A B$ effect.

The $\mathrm{AB}$ effect is a $\mathrm{QM}$ wave function phase shift resulting from a charged particle in vector potential, predicted by the Schrödinger equation and evident in interference patterns of electrons in two-slit experiments (see Fig. 1). What is remarkable is that the phase shift occurs in the absence of a magnetic field (B). In general, student first learn the vector potential (A) as a purely

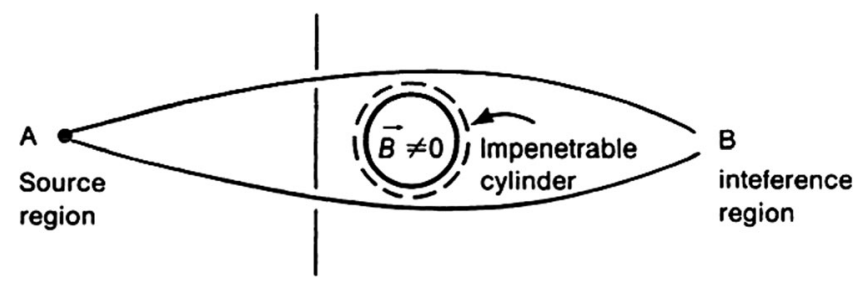

FIG. 1. Double-slit experiment illustrating the AB effect. Given the possible electron paths from source at $A$ to detector at $B$, the resulting interference pattern changes as the $\mathbf{B}$ in the impenetrable cylinder is turned on or off. Figure reproduced from J. J. Sakurai's Modern Quantum Mechanics. mathematical construct for finding the physically significant $\mathbf{B}$. The AB effect shows the vector potential to have direct physical significance itself. There is no classical analog of the $\mathrm{AB}$ effect.

The interview begins with a question about what has captured Bailey's attention in class, anything that is "weird or interesting or surprising." He brings up quantum interference, and the $\mathrm{AB}$ effect in particular.

Bailey describes the effect by introducing the $\mathbf{B}$ and how it is the curl of A. He says for the AB effect to happen, the A needs to be different valued in space along the electron's possible trajectories, resulting in a difference in phase between them. He notes the phase difference depends solely on the magnetic flux through the enclosed loop bounded by the paths. Bailey insinuates that by itself, this behavior is not surprising: a magnetic flux through a loop can effect a phase shift. But, he goes on to say, it gets interesting when the field is localized:

1 Bailey: If we instead localized our $\mathbf{B}$, if we've

2 just got a solenoid standing here and

3 we're letting the particles sort of go

4 around that, in that arrangement we have

5 a $\mathbf{B}$ in the center of the solenoid, but

6 we have no $\mathbf{B}$ outside of that. So it's

7 this isolated local field and the area

8 through which the particles are passing

9 is void of any $\mathbf{B}$, so they're not

10 experiencing any $\mathbf{B}$ as they travel there,

11 you know, can't be any work done on them

12 by this $\mathbf{B}$, um, but there is the flux

13 right in that sort of path

14 Vesal: There's flux of

15 Bailey: There's still magnetic flux in that

16 Vesal: within the solenoid

17 Bailey: larger, well yeah, within the solenoid,

18 and therefore within the path. Um,

19 [smiling] (uh oh) and therefore, we see

20 this interference pattern that we'd

21 expect, if that same amount of magnetic

22 flux were spread out through the entire

23 thing.

Bailey explains the $\mathrm{AB}$ effect and describes its unexpected nature. Vesal then further probes his thinking about it:

24 Vesal: So the weirdness enters because there is

25 no $\mathbf{B}$ that is interacting with the

26 particles.

27 Bailey: Yeah, it's a super localized $\mathbf{B}$, and yet

28 its effects are seen on particles in

29 which pass through areas that are, um,

30 well that don't have any $\mathbf{B}$.

31 Vesal: So what does that lead you to? I mean,

32 what do you think about that?

33 Bailey: [laughing] I think it's awesome! Um, 34 so, yeah I think it's cool because a lot 
35 of times we come to these conclusions in

36 QM and we can sort of, um, you know, take

37 some limit of them and see that you know

38 we would expect this classically also,

39 um, but an effect like this is just

40 purely a QM effect and as far as I can

41 tell there isn't um, you know there isn't

42 any sort of classical way to come to

43 terms with that.

44 Vesal: So when you say QM effect, what do you 45 mean?

46 Bailey: I mean that we, I guess I mean that we

47 can only explain it using QM, um, yeah. I

48 think that's the simplest way to phrase

49 it. And I guess, so one of the things

50 that's interesting so you asked me

51 earlier [...] about sort of which of

52 these things are real and I'm wondering

53 if this effect sort of legitimizes the A

54 as a more real thing in my mind.

55 Vesal: Why do you think, why do you say that?

56 Bailey: But I don't know if I actually-well,

57 so I was thinking cause I know that the

58 sort of derivation to get to this

59 conclusion um, was, I mean depended on

60 that, um, [starting to look through

61 notebook] I'm sort of blanking on, but,

62 let's see if I can quickly pick up

63 anything about this...[looks through

64 notebook for a minute] So the thing is

65 that I don't, we were sort of slogging

66 through a lot of math to get to this, and

67 um, you know, we go through a lot of

68 heavy math to get to any single

69 conclusion that we get to, it seems, um,

70 but you know, we sort of, we ended at

71 this place and it was like, well wait,

72 and you know you sort of looked at the

73 fact that it's, it's just this localized

74 field can cause a nonlocal effect, um,

75 and that's when you realize that you

76 should pay more attention to the math

77 that lead you there [laughing] — wait I

78 need to understand that! That's amazing!

79 [looking at notebook]

80 Vesal: So, in your mind, the derivation would

81 have helped explain why?

82 Bailey: So, I think so because this isn't um, I

83 mean I can tell you that this occurs and

84 I can tell you that we see it

85 experimentally and can predict it, but I

86 right now cannot tell you why it occurs.

Bailey's excitement about the strangeness of the $A B$ effect is tempered only by what he feels is an unsatisfactory attempt at accounting for it: either a localized (magnetic) field is having a nonlocal effect (interference pattern) or the vector potential has some physical significance. ${ }^{3}$ Most relevant is that in his desire to understand the phenomenon and provide a better explanation, Bailey says he must go back and examine the dense mathematical derivation leading to the "amazing" conclusion. Bailey is surprised by the nonlocal effect of the $\mathbf{B}$, which leads him to expect that the mathematics has physically meaningful substance to it, and that the weirdness of the effect can be understood by following the derivation.

Later in the interview, Bailey reflects on this view of sense making more explicitly:

87 Bailey: Yeah, I mean the way, it's too bad

88 because the way it sort of, I mean it

89 gets at one of the things that makes QM

90 difficult is that we see these effects

91 that seem to, um, that seem to be almost

92 caused by just the way that the

93 mathematics work out and not something

94 that we can, um, not something that we

95 can qualitatively justify, um, which, one

96 makes them harder to explain, and two

97 requires a deeper understanding of how

98 the math did work out, um, which I'm

99 still trying to build.

Thus in this episode Bailey believes he needs "deeper understanding" of the mathematics, in order to make sense of the $\mathrm{AB}$ effect. He frames learning $\mathrm{QM}$ to involve drawing physical meaning from the derivation, in line with the epistemic game Tuminaro and Redish [96] called Mapping mathematics to meaning. There is evidence of a similar framing in Bailey's approach broadly across his interviews, as well as in Bailey's explicit comments, including during the first interview, of how mathematics and life experience relate to sense making in QM:

100 Bailey: When you're first learning about it

101 [QM], you don't have sort of empirical

102 proof that you've been exposed to growing

103 up, and so you don't therefore, um, or at

104 least I didn't therefore have the same

105 sense of like immediate understanding. It

106 was more of a, um, you know, let's just

107 be around it, let's try to like come at

108 it from the mathematical side instead of

109 the-you know, there's much less having

110 like the life experience and mathematical

111 sides come meet [motioning hands to the

112 middle], it's more like the mathematical

113 side really reaches most of the way, and

\footnotetext{
${ }^{3}$ Bailey's thinking here has a parallel in physics history: The vector potential was first constructed assuming the existence of mathematical symmetry. That is, there is a potential for the electric field, so what would it be for the magnetic field? The vector potential was devised, but it was not thought to have physical significance.
} 
114 then you have some observations from the

115 other side that sort of help you connect

116 those, um.

Bailey talks of mathematics as more consequential for sense making in QM as compared to in classical mechanics, where life experience and mathematics are more mutually reinforcing. That is, understanding the macroworld involves correlating everyday experience with classical theory, whereas understanding the subatomic realm mostly comes from examining the mathematical framework of quantum theory (with observation playing a reduced role in that development).

\section{Studying scattering: Mathematics as distinct from conceptual meaning}

In the weeks before the fourth interview of the second semester, the class was covering scattering theory. Here we present Bailey's describing a homework problem to reproduce a figure in the textbook related to scattering.

Scattering theory, in essence, is about understanding and calculating how particles deflect as a result of a collision or interaction: Shoot pellets at a bowling ball, or a beam of electrons at a nucleus, and they will "bounce off" at various angles. QM scattering, unlike classical, is nondeterministic, calculating the probability of scattering into a given angle (or small range of angles). The probability is generally expressed as a cross-sectional area, an abstraction of a classical view of the phenomenon: The larger the area, the greater the chance of a particle "hitting it" (see Fig. 2).

We can think of a quantum scattering process as a system transitioning from an initial to a final state that is effected by a perturbation (the initial and final states encode angle information). The conceptualization of the interaction itself is very different between classical and QM. Classically, the precise initial state of a particle (a pellet) and specification of the scattering object (bowling ball) determines what takes place. In $\mathrm{QM}$, the matrix

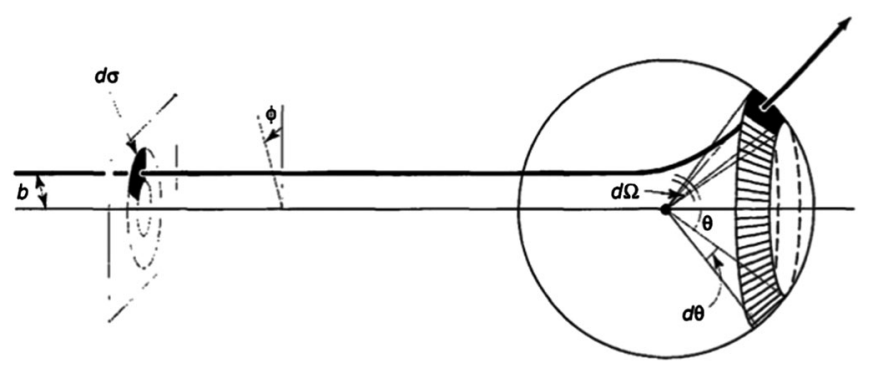

FIG. 2. A central problem in scattering theory is to characterize the probability of scattering in a given direction, essentially the scattering amplitude $[f(k, \theta)]$, which depends on the incident cross section $(d \sigma)$ as compared to the solid angle $(d \Omega)$ it scatters into. Figure reproduced from D. J. Griffiths Introduction to Quantum Mechanics. element $\left\langle f\left|\hat{H}_{\text {pert }}\right| i\right\rangle$ provides the probability amplitude of the transition from the initial state to the final state as a result of the interaction.

Beginning the discussion, Vesal asks Bailey to work through any problem that has been interesting to him. Bailey starts to describe one in which students had to reproduce a figure in their textbook related to resonance scattering. Before getting into it, he comments that he has experienced scattering theory as "very formula based and not necessarily...conceptually super grounded." Indeed, in his initial attempt at describing it he resorts to listing pertinent variables rather than offering an explanation.

Moments later he has trouble describing what happens when a wave interacts with a potential, first saying that he does not really know what has motivated the derivation of the "whole list of formulas" he has in his notes, and then saying "it seems like there should be a next logical step here where basically we just draw this line closer [referring to two entities on the verge of colliding] and then talk about like, ok, how did these interact! Right, but I feel like in my mind I don't have that progression super clear. And instead we just sort of skip right from this to define this thing that's [writing] $f(k, \theta)$, and after that we also have this $\delta$, and some like cross-sectional area..." Bailey's frustration stems from not knowing how these variables that populate the formulas he has encountered fit into an explanation of what physically happens during a scattering event. Bailey then tries to think about what the variables in the problem represent,

117 Bailey: We sort of decide that there are some 118 important, you know, basically variables 119 at which we need to look to understand 120 what's going on. And so, I'm trying to

121 think of physically what $f(k, \theta)$

122 represents, but I'm drawing a blank. So,

123 we talk about... Um, see, that's the

124 problem with this stuff. I go to explain

125 it, and I find that the only way I can

126 explain it is by writing down equations

127 then trying to talk about what those

128 mean, and I feel like that's a terrible 129 way to understand something.

130 Vesal: Why is that?

131 Bailey: You know, like you want to

132 be able to talk about it qualitatively, (right,

133 right) you want to have both of those

134 sides, you want to be able to do both,

135 but it shouldn't, I don't know, I don't

136 like the reliance on these equations that

137 is happening on this piece of paper right

138 now. (understood) Basically just me

139 complaining about my lack of

140 understanding with this stuff at the

141 moment, um... 
Here Bailey expresses frustration that he needs to rely on equations to explain the meaning of the differential crosssection. He expects to offer a conceptual explanation, alongside any mathematical description, but realizes he cannot. This prompts him to complain about how typical physics instruction presents equations without conceptual explanation, when it should be "it's very simple to say okay, let's put the equations aside and let's talk about what happens."

142 Bailey: You can introduce equations to help you

143 along and to like, to like, I guess

144 bolster your foundation, but you don't

145 necessarily have to rely on them to get

146 to each next step, it's, it's sort of

147 logic and observation based. [...]

148 Vesal: So right now you're debating whether

149 it's possible to talk about some of these

150 [QM] ideas without-

151 Bailey: an equation driving us, right, I think

152 it needs to be there somewhere, but do we

153 need to necessarily start with, like,

154 here's an equation, let's talk about what that

155 means. Or can we get there through

156 you know almost like thought experiment,

157 thinking about like, OK, well, what

158 happens in this sort of situation and

159 then you just sort of bolster our

160 conclusions with an equation after the

161 fact.

Thus Bailey describes missing in QM what he experiences in introductory physics, of ideas beginning with "sort of logic and observation," with equations coming along to "bolster your foundation," but not as the starting point.

Bailey had made a few attempts at constructing a conceptual explanation just prior to the exchange above. For example, when he cannot explain the interaction cross section within the context of QM, he turns to classical ideas using trajectories of balls and drawing a graph of cross section vs wave number as constant. Even though he knows the classical ideas are far removed from quantum behavior, he brings them because he has a conceptual grasp of those ideas, and so he expects them to serve as a starting point for discussion.

Shortly thereafter, Bailey took another shot at putting together his classical expectations with quantum phenomena: he constructed what he called a semiclassical account of scattering as a way to animate his thinking about variables that lacked meaning for him. In his account, an incident object is represented by an incoming probability distribution of possible states, each of which individually interacts with the potential and scatters (see Fig. 3). By mapping each scattered state deterministically back to the original one that gave rise to it, and weighting it according to that probability, he finds the overall outcome of the interaction. But Bailey realizes his explanation has

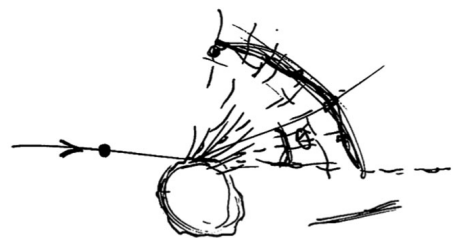

FIG. 3. Bailey constructs what he calls a "semiclassical" explanation of scattering that is represented in this figure. On the left side there is an incoming electron that he represents as a distribution of states. He says that a particular state from the distribution he has drawn on the left side interacts with a scattering potential, which he represents as a circle on the right side. Each possible electron state scatters in a particular way, resulting in one trajectory among the several he has drawn that originate at the scatterer. The final state of the electron is rebuilt by combining all of those possible trajectories.

limitations and boils down to a "weighted average over a series of probabilities," which he considers too simplistic and essentially classical ${ }^{4}$ (one that is not "terribly interesting" and "lame", ).

These examples show how Bailey is inclined to first illustrate scattering conceptually, and then expects to use mathematics to make that thinking more precise (i.e., doing it in a "rigorous mathematical way"). Thus, Bailey expects the mathematics to follow conceptual understanding in line with the game Tuminaro and Redish [96] called Mapping meaning to mathematics. In Sec. IV, we propose a theoretical account of these different framings. Bailey himself offered the beginning of an analysis, when, at the end of the episode, he reflects on his thinking:

162 Bailey: For a one-on-one conversation like

163 this, to me it doesn't make sense to be

164 focusing on equations, right, I'd rather

165 talk about concepts and just sort of

166 being able to throw down an equation and

167 say like, "OK, this thing that we've

168 been talking about, here's something we

169 can look at that supports this side of

170 it, and look, there's this other

171 interesting observation that we can make

\footnotetext{
${ }^{4}$ Someone might argue that Bailey's prescription to calculate the scattering probability may be originating from the mathematical structure of scattering theory, since a kind of "weighted average over a series of probabilities" appears in Fermi's golden rule. While it is clear he is not thinking about a density of states as a weighting function or the scatterer as perturbing potential that facilitates a transition of a given probability, it is at least plausible that an expression of that kind occurred to him because of exposure to the theory. Regardless, connecting to and deriving meaning from the mathematics is not an explicit commitment for him as it was during the $\mathrm{AB}$ episode.

${ }^{5}$ It is worth mentioning that while Bailey considers his explanation a "lame attempt," he has every intention of refining it as he says immediately after "it's something that we'll be able to build on as we go."
} 
172 about this," um, and I was about to say

173 that, that that's something you'd

174 especially want to do in a conversation

175 like this, you know, as opposed to being

176 at a blackboard in front of a class of

177 students, but then I realized that that

178 might not be the case maybe, in both

179 cases it's better to be able to motivate

180 it conceptually. But, um...yeah, so

181 that's a long aside about how I would

182 like to be talking about this. I lack the

183 tools for the moment.

\section{ANALYSIS: AN ACCOUNT OF BAILEY'S MULTIPLE FRAMINGS}

In the previous section, we presented evidence of how Bailey framed learning in QM, in particular with respect to mathematics. Working to understand the $\mathrm{AB}$ effect, Bailey described and approached the mathematics as a basis for forming conceptual understanding; this characterized most of his work over the year. Working to understand scattering, however, Bailey described and approached the mathematics as secondary, as enhancing or building from conceptual understanding but not as a basis.

In this section, we offer a theoretical interpretation of this variation, in line with dynamic accounts of epistemologies we reviewed in the introduction, both in general $[88,90]$, and specifically with respect to QM [58,59]. We base our interpretation on Thelen's and Smith's [110] studies of development in young children. They have modeled infants' and toddlers' learning to reach and to walk, and more recently learning words [111], as "soft assemblies" of dynamic systems (Kugler and Turvey [112] cited in Thelen and Smith [110]). Their models have shown success in predicting outcomes in novel situations.

Here we consider Bailey's different views and approaches in the episodes above as the dynamics of systems that extend beyond him as an individual. Thinking and behavior patterns appear from relations among resources we attribute to Bailey, including epistemological resources as we discussed above as well as conceptual, affective, and social, in interaction with the features of the context, including the subject matter, the QM course, as well as the interview itself. That is, we interpret Bailey's framings of mathematics as soft-assemblies. In what follows we suggest how parts of these systems may have contributed to those assemblies.

\section{A. Instructional approaches}

There was evidence across subjects of contrasting experiences in the two courses, from first to second semester. Specifically, Bailey and other subjects spoke of the professor in the first course welcoming and responding to student questions, asking students to explain the meaning behind equations and to work out next steps for problems and derivations. That professor also made efforts to connect real world applications to abstract formalism. In contrast, Bailey and other subjects spoke of the second course as a traditional lecture, focused on the mathematical derivations, with little discussion of conceptual interpretations or applications. In fact, Bailey at one point complained that the course's approach to perturbation theory was a "graduate level plug-n-chug exercise."

\section{B. Conceptual affordances of the subject matter}

The $A B$ effect is striking in particular for its contrast with physical intuition from classical mechanics and experience: It is an electromagnetic influence on physical phenomena in the absence of electric or magnetic fields. For a student interested to understand the phenomenon, it is hard to think where else to look for insight other than the mathematics.

Scattering theory, in contrast, has a clear classical analog, manifest in the notion of a cross section, expressed and visualized as an area the particle enters. For a student interested, classical ideas and experience are obvious places to look for help in understanding. Meanwhile, it is important to note, the mathematics of QM scattering theory diverge significantly from classical ideas, in particular in matrix element calculation of probability amplitudes.

\section{The interview itself}

There is clear evidence the interview context itself was involved in the dynamics, in Bailey's reflecting on what is appropriate in a "conversation like this," at the end of the scattering episode. ${ }^{6} \mathrm{He}$ saw the interview as a place to converse about ideas, and to him, writing equations divorced from sensible reasoning violates the conversational norm. That is, Bailey feels accountable to explaining his understanding, in contrast to his experience of the second semester course.

\section{Bailey's resources}

Finally, and necessarily for this account, Bailey has a rich, extensive variety of resources for thinking about and experiencing the world, from physical phenomena to social interactions to epistemic activities. He has, for example, a sense of a plug-n-chug exercise, which comes into play in his experience of the second course, as distinct from a pursuit of understanding; conceptual resources for

\footnotetext{
${ }^{6}$ Bailey, in fact, went on to express how the interview might have an effect on his learning, in the second course: "It's going to be much more interesting reading through my notes now... I think I'm just having the opportunity to talk through this stuff is more, cause it always does in these sessions, it makes me more aware of what my actual questions are, and so I think I'll be, I think I'll be much better poised to understand what I'm doing when I go back and read through things... Before I sort of just like let it pass and just wrote down equations."
} 
understanding classical dynamics and everyday experience of collisions, which we have suggested come into play as he tries to make sense of scattering; a sense of meaningful discussion to understand "a conversation like this."

It is important to distinguish related meanings of stability in discussing these dynamics. In one sense, we speak of Bailey as "having resources," attributing them to him as available for activation. We attribute a resource in this way to an individual when we see or expect it to arise across a variety of contexts. For example, we can expect a sense of plug-n-chug arises in multiple situations for Bailey, and we can think of that sense as among his epistemological resources.

We also speak of a pattern of activation as stable within a particular context, but possibly only in that context. For example, when Bailey says "in a one-on-one conversation like this," it is evidence he has a sense of what is taking place, a framing, but it is hard to know whether that particular sense comes up across other contexts. Quite likely, he has not experienced an interview precisely like this before, but he has experienced other sorts of interactions that help him frame what is taking place here. Of course, if Bailey participates in another study that involves interviewing him about his reasoning in a course, he will come to understand it more quickly.

Of course, Bailey comes to graduate QM with a great deal of prior experience learning physics, and it is clearly involved in how he experiences QM, in both courses. Across these interviews, we have seen evidence of two ways of framing the role of mathematics in learning physics, invoking the epistemic games of Mapping mathematics to meaning and Mapping meaning to mathematics [96]. We think of these different epistemic games as resources available for his activation. The stable pattern evident in the $\mathrm{AB}$ episode involves the former, and in the scattering example involves the latter.

\section{FINDINGS AND IMPLICATIONS}

Here we summarize findings from our analyses of Bailey's epistemology, and we discuss their implications for further work.

\section{A. Variation across episodes}

Our first claim is that Bailey's epistemology was different across the two episodes with respect to the roles of physical experience and mathematics. Studying the AB effect, he showed and expressed an expectation that mathematical derivations convey conceptual substance; studying scattering theory, he showed and expressed an expectation that conceptual substance comes out of "logic and observation," not from math. In terms from Tuminaro and Redish [96], he varied in particular in his activation of Mapping mathematics to meaning.
We did not, in either interview, witness Bailey's arrival at understanding either the $\mathrm{AB}$ effect or scattering. In the first, however, we gave evidence of productive engagement: He was interested and making progress, recognizing key features of the phenomenon and working to grapple with their implications. He made at least one meaningful inference from the mathematics, that it shows there is a physical effect on the electron without a local magnetic field, considering that a magnetic field might have a "nonlocal" effect. In the second, he felt stuck, that he "lacked the tools" to make sense of the phenomenon, and frustrated that the course was only providing mathematics, not understanding.

Bailey's variation in this respect suggests it is an area of ongoing development for him. In general, Dini [65] shows, Bailey looked for meaning in the mathematics. The evidence here shows that he was not stable in that framing to maintain it in all contexts of his QM courses.

\section{B. Stabilities across episodes}

There were also aspects of Bailey's epistemology that were consistent across the episodes.

First, he showed a commitment throughout to sensemaking and coherence. If in moments during his work in the course he varied from that, on recognizing that he spoke of it as a lapse: Bailey was looking for understanding, and he was not satisfied simply to learn the steps of, for example, how to calculate a scattering cross section. That is, there was pervasive evidence of his working to connect the mathematics with meaningful understanding of the physical world; the variation was in how he engaged in that effort.

Second, and related, across these episodes and his interviews more generally, Bailey engaged explicitly in considering epistemology. That is, he was articulate and reflective in his commitment to sense-making and coherence. He did not simply abandon everyday experience and physical intuition, on recognizing contradictions; it seemed rather to prompt him to examine the nature of knowing.

\section{Possibilities for instruction and further research}

This is, of course, only a single case, and of an especially successful student. ${ }^{7}$ What we can take from it specifically can only be conjectural, regarding possibly useful new directions for instruction and for research.

Our findings with respect to Bailey supports arguments for greater attention and emphasis to matters of epistemology in QM instruction [24,99]. They also support views of student epistemologies as dynamic, varying with context in

\footnotetext{
${ }^{7}$ Dini [65] presents data from other students that shows Bailey was not unique in these respects. Of the eight graduate students and five undergraduates in the larger study, Bailey was one of the four who were most successful, by our sense from interviews and consistent with their course grades.
} 
interaction with features of instruction, in particular with respect to the role of mathematics [59,96] and, quite specifically, Tuminaro's and Redish's identification of Mapping mathematics to meaning and Mapping meaning to mathematics as distinct epistemic activities and epistemological framings.

For Bailey and other students successful with classical theories, learning physics had always involved putting common sense into equations, as well as modify[ing] common sense based on new ideas that derive from those equations [57]. In Bailey's terms, "the life experience and mathematical sides come meet" in the middle. These students come to a stability in understanding knowledge in physics as coherent and conceptual, the mathematics speaking with and about that understanding. We suggest that, for Bailey, that commitment was essential to his success in learning QM, but that it needed adjustment in two respects.

In classical physics, finding a contradiction with one part of common sense, learners can shop for different connections to other parts of common sense. Thus, for example, students can come to understand a table surface as pushing by tapping into resources for understanding springs [113,114]. Studying QM, Bailey continued to seek coherence with respect to his life experience, but the reconciliation had to be epistemological. It was not sufficient for him simply to disregard his classical ideas and experience of the world; he needed to grapple explicitly with the shift in applicability of that knowledge.

We suspect the same is true for other students, that such grappling is essential for them to maintain their productive commitment to meaning and coherence, so that learning physics does not become a matter of memorizing calculation techniques provided by authority. In this, we support prior calls for greater attention and emphasis on epistemology in QM instruction, in particular to help students navigate the shift from what had been so successful for them in studying classical mechanics:

Everyday thinking can be misleading in quantum physics, but that is not a sufficient argument for the wholesale abandonment of productive epistemological tools. What is important is that students understand the limitations of these intuitive conceptions, and where they might lead them astray (p. 12) [24].

Note that this call is not for "coverage" or for arrival as a particular, "correct" epistemology of QM, which remains controversial. Rather, it is for courses explicitly to support students in their sense making at this level. This would mean a shift for instructors who frame teaching as conveying clear information to instruction as supporting students' disciplinary inquiry.

Finally, our findings for Bailey suggest research to consider another emphasis. While he was stable in framing physics as sensible, he was not stable in seeing mathematics as expressing conceptual substance; he did not invoke the epistemic game of Mapping mathematics to meaning [96] in contexts when it could have served him. This needs further study. Perhaps this needs greater attention as a specific instructional target in earlier courses, anticipating the need in QM.

\section{ACKNOWLEDGMENTS}

The authors wish to thank Krzysztof Sliwa, Roger Tobin, and Hugh Gallagher for useful discussions, as well as Ayush Gupta, Ben Dreyfus, Erin Sohr, Brandon Johnson, Andy Elby, and Jessica Hoehn for feedback on our analysis. The first author is grateful to the John F. Burlingame Graduate Fellowship in partly supporting the completion of this work.
[1] S. B. McKagan and C. E. Wieman, Exploring student understanding of energy through the quantum mechanics conceptual survey, AIP Conf. Proc. 818, 65 (2006).

[2] J. T. Morgan and M. C. Wittmann, Examining the evolution of student ideas about quantum tunneling, AIP Conf. Proc. 818, 73 (2006).

[3] M. C. Wittmann, J. T. Morgan, and L. Bao, Addressing student models of energy loss in quantum tunnelling, Eur. J. Phys. 26, 939 (2005).

[4] G. Zhu and C. Singh, Surveying students' understanding of quantum mechanics in one spatial dimension, Am. J. Phys. 80, 252 (2012).

[5] E. Marshman and C. Singh, Investigating student difficulties with time dependence of expectation values in quantum mechanics, in Proceedings of the 2013 Physics Education Research Conference, Portland, OR, 2013 edited by P. Engelhardt, A. Churukian, and D. Jones (2014), pp. 245-248.

[6] P. J. Emigh, G. Passante, and P. S. Shaffer, Student understanding of time dependence in quantum mechanics, Phys. Rev. ST Phys. Educ. Res. 11, 020112 (2015).

[7] M. Ayene, J. Kriek, and B. Damtie, Wave-particle duality and uncertainty principle: Phenomenographic categories of description of tertiary physics students' depictions, Phys. Rev. ST Phys. Educ. Res. 7, 020113 (2011).

[8] K. Mannila and I. T. Koponen, and J. A. Niskanen, Building a picture of students' conceptions of waveand particle-like properties of quantum entities, Eur. J. Phys. 23, 45 (2002). 
[9] S. Vokos, Student understanding of the wave nature of matter: Diffraction and interference of particles, Am. J. Phys. 68, S42 (2000).

[10] R. V. Olsen, Introducing quantum mechanics in the upper secondary school: A study in Norway, Int. J. Sci. Educ. 24, 565 (2002).

[11] G. Zhu and C. Singh, Improving students understanding of quantum measurement. I. Investigation of difficulties, Phys. Rev. ST Phys. Educ. Res. 8, 010117 (2012).

[12] G. Zhu and C. Singh, Improving students understanding of quantum measurement. II. Development of researchbased learning tools, Phys. Rev. ST Phys. Educ. Res. 8, 010118 (2012).

[13] H. Sadaghiani and L. Bao, Student difficulties in understanding probability in quantum mechanics, AIP Conf. Proc. 818, 61 (2006).

[14] C. Singh, Student understanding of quantum mechanics, Am. J. Phys. 69, 885 (2001).

[15] E. Gire and C. Manogue, Making sense of quantum operators, eigenstates and quantum measurements, AIP Conf. Proc. 1413, 195 (2012).

[16] G. Zhu and C. Singh, Students' understanding of Stern Gerlach experiment, AIP Conf. Proc. 1179, 309 (2009).

[17] C. Singh, Student difficulties with quantum mechanics formalism, AIP Conf. Proc. 883, 185 (2007).

[18] C. Singh and E. Marshman, Investigating student difficulties with dirac notation, in Proceedings of the 2013 Physics Education Research Conference, Portland, OR, 2013 edited by A. Churukian, P. Engelhardt, and D. Jones (2014), pp. 345-348.

[19] L. D. Carr and S. B. McKagan, Graduate quantum mechanics reform, Am. J. Phys. 77, 308 (2009).

[20] S. J. Pollock, S. V. Chasteen, and M. Dubson, The use of concept tests and peer instruction in upper-division physics, AIP Conf. Proc. 1289, 261 (2010).

[21] S. M. Gómez Puente and H. J. M. Swagten, Designing learning environments to teach interactive Quantum Physics, Eur. J. Eng. Educ., 37, 448 (2012).

[22] C. Manogue, E. Gire, D. McIntyre, and J. Tate, Representations for a spins-first approach to quantum mechanics, AIP Conf. Proc. 1413, 55 (2012).

[23] D. Demaree, Applying ISLE ideas to active engagement in the spins paradigm, AIP Conf. Proc. 1289, 17 (2010).

[24] C. Baily and N. D. Finkelstein, Teaching quantum interpretations: Revisiting the goals and practices of introductory quantum physics courses, Phys. Rev. ST Phys. Educ. Res. 11, 020124 (2015).

[25] R. Müller and H. Wiesner, Teaching quantum mechanics on an introductory level, Am. J. Phys. 70, 200 (2002).

[26] M. C. Wittmann, R. N. Steinberg, and E. F. Redish, Investigating student understanding of quantum physics: Spontaneous models of conductivity, Am. J. Phys. 70, 218 (2002).

[27] L. T. Escalada, N. S. Rebello, and D. A. Zollman, Student explorations of quantum effects in LEDs and luminescent devices, Phys. Teach. 42, 173 (2004).

[28] L. T. Escalada, Ph.D. thesis, Kansas State University 1997.

[29] M. C. Wittmann, J. T. Morgan, and R. E. Feeley, Laboratory-tutorial activities for teaching probability, Phys. Rev. ST Phys. Educ. Res. 2, 020104 (2006).
[30] L. Bao and E. F. Redish, Understanding probabilistic interpretations of physical systems: A prerequisite to learning quantum physics, Am. J. Phys. 70, 210 (2002).

[31] S. B. McKagan, K. K. Perkins, and C. E. Wieman, Why we should teach the Bohr model and how to teach it effectively, Phys. Rev. ST Phys. Educ. Res. 4, 010103 (2008).

[32] P. Jolly, D. Zollman, and N. S. Rebello, Visualizing motion in potential wells, Am. J. Phys. 66, 57 (1998).

[33] S. Klassen, The photoelectric effect: Reconstructing the story for the physics classroom, Sci. Educ. 20, 719 (2011)

[34] M. Niaz, S. Klassen, B. McMillan, and D. Metz, Reconstruction of the history of the photoelectric effect and its implications for general physics textbooks, Sci. Educ. (2010).

[35] M. A. Asikainen and P. E. Hirvonen, A study of pre-and inservice physics teachers' understanding of photoelectric phenomenon as part of the development of a researchbased quantum physics course, Am. J. Phys. 77, 658 (2009).

[36] S. B. McKagan, W. Handley, and K. K. Perkins, A research-based curriculum for teaching the photoelectric effect, Am. J. Phys. 77, 87 (2009).

[37] R. N. Steinberg, G. E. Oberem, and L. C. McDermott, Development of a computer-based tutorial on the photoelectric effect, Am. J. Phys. 64, 1370 (1996).

[38] G. Zhu and C. Singh, Improving students' understanding of quantum mechanics via the Stern-Gerlach experiment, Am. J. Phys. 79, 499 (2011).

[39] G. Zhu and C. Singh, Improving student understanding of addition of angular momentum in quantum mechanics, Phys. Rev. ST Phys. Educ. Res. 9, 010101 (2013).

[40] M. Belloni and W. Christian, Physlets for quantum mechanics, Comput. Sci. Eng. 5, 90 (2003).

[41] S. B. McKagan, K. K. Perkins, M. Dubson, and C. Malley, Developing and researching PhET simulations for teaching quantum mechanics, Am. J. Phys. 76, 406 (2008)

[42] L. T. Escalada, An investigation on the impact of implementing visual quantum mechanics on student learning and student instructor beliefs, in Proceedings of the 2001 Physics Education Research Conference, National Physics Education Research Conference edited by S. Franklin, J. Marx, and K. Cummings (2001), pp. 21-24

[43] A. Kohnle, M. Douglass, and T. J. Edwards, Developing and evaluating animations for teaching quantum mechanics concepts, Eur. J. Phys. 31, 1441 (2010).

[44] A. Kohnle, D. Cassettari, T. J. Edwards, C. Ferguson, A. D. Gillies, C. A. Hooley, N. Korolkova, J. Llama, and B. D. Sinclair, A new multimedia resource for teaching quantum mechanics concepts, Am. J. Phys. 80, 148 (2012).

[45] I. Kohnle, A. Bozhinova, and D. Browne, A new introductory quantum mechanics curriculum, Eur. J. Phys. 35, 015001 (2014).

[46] A. Kohnle, C. Baily, and S. Ruby, Investigating the influence of visualization on student understanding of quantum superposition, in PERC Proceedings Minneapolis, MN, 2014 edited by P. V. Engelhardt, A. D. Churukian, and D. L. Jones (2014). 
[47] C. Singh, Student understanding of quantum mechanics at the beginning of graduate instruction, Am. J. Phys. 76, 277 (2008).

[48] C. Singh and G. Zhu, Improving students' understanding of quantum mechanics by using peer instruction tools, AIP Conf. Proc. 1413, 77 (2012).

[49] E. Cataloglu and R. W. Robinett, Testing the development of student conceptual and visualization understanding in quantum mechanics through the undergraduate career, Am. J. Phys. 70, 238 (2002).

[50] S. Wuttiprom, M. D. Sharma, I. D. Johnston, R. Chitaree, and C. Soankwan, Development and Use of a Conceptual Survey in Introductory Quantum Physics, Int. J. Sci. Educ. 31, 631 (2009).

[51] S. Goldhaber, S. Pollock, M. Dubson, and P. Beale, Transforming upper-division quantum mechanics: Learning goals and assessment, in Proceedings of the 2009 Physics Education Research Conference (AIP, Melville, NY, 2009), Vol. 1179, p. 145-148.

[52] L. Deslauriers and C. Wieman, Learning and retention of quantum concepts with different teaching methods, Phys. Rev. ST Phys. Educ. Res. 7, 010101 (2011).

[53] S. B. McKagan, K. K. Perkins, and C. Wieman, Design and validation of the Quantum Mechanics Conceptual Survey, Phys. Rev. ST Phys. Educ. Res. 6, 020121 (2010).

[54] H. R. Sadaghiani and S. J. Pollock, Quantum mechanics concept assessment: Development and validation study, Phys. Rev. ST Phys. Educ. Res. 11, 010110 (2015).

[55] R. K. Thornton and D. R. Sokoloff, Assessing student learning of Newton's laws: The force and motion conceptual evaluation and the evaluation of active learning laboratory and lecture curricula, Am. J. Phys. 66, 338 (1998).

[56] D. Hestenes, M. Wells, and G. Swackhamer, Force concept inventory, Phys. Teach. 30, 141 (1992).

[57] D. Hammer, Epistemological beliefs in introductory physics, Cognit. Instr. 12, 151 (1994).

[58] C. Baily and N. D. Finkelstein, Development of quantum perspectives in modern physics, Phys. Rev. ST Phys. Educ. Res. 5, 010106 (2009).

[59] T. Bing and E. F. Redish, Epistemic complexity and the journeyman-expert transition, Phys. Rev. ST Phys. Educ. Res. 8, 010105 (2012).

[60] C. Singh and E. Marshman, Review of student difficulties in upper-level quantum mechanics, Phys. Rev. ST Phys. Educ. Res. 11, 020117 (2015).

[61] B. Modir and E. C. Sayre, 'Pulling out' as a procedural resource when solving partial differential equations, arXiv:1607.07493.

[62] A. Hobson, Response to "The real scandal of quantum mechanics", By Richard Conn Hennry, Am. J. Phys. 77, 869 (2009); Am. J. Phys. 77, 870 (2009).

[63] C. Sommer, Another survey of foundational attitudes towards quantum mechanics, arXiv:1303.2719v1.

[64] A. Einstein, Physics and reality, J. Franklin Inst. 221, 349 (1936).

[65] V. Dini, Ph.D. thesis, Tufts University, 2017.

[66] A. A. diSessa, Learning about knowing, in Children and computers: New directions for child development edited by E. Klein (Jossey-Bass, San Francisco, 1985), Vol. 28, pp. 97-124.
[67] P. W. Hewson, Epistemological commitments in the learning of science: Examples from dynamics, Eur. J. Sci. Educ. 7, 163 (1985).

[68] A. Elby, Helping physics students learn how to learn, Am. J. Phys. 69, S54 (2001).

[69] J. Leach, R. Millar, J. Ryder, and M. G. Séré, Epistemological understanding in science learning: The consistency of representations across contexts, Learn. Instr. 10, 497 (2000).

[70] C. J. Linder, Is teacher-reflected epistemology a source of conceptual difficulty in physics?, Int. J. Sci. Educ. 14, 111 (1992).

[71] D. B. May and E. Etkina, College physics students? Epistemological self-reflection and its relationship to conceptual learning, Am. J. Phys. 70, 1249 (2002).

[72] W. M. Roth and A. Roychoudhury, Physics students' epistemologies and views about knowing and learning, J. Res. Sci. Teach. 31, 5 (1994).

[73] E. F. Redish, J. M. Saul, and R. N. Steinberg, Student expectations in introductory physics, Am. J. Phys. 66, 212 (1998).

[74] W. K. Adams, K. Perkins, and N. S. Podolefsky, New instrument for measuring student beliefs about physics and learning physics: The colorado learning attitudes about science survey, Phys. Rev. ST Phys. Educ. Res. 2, 010101 (2006).

[75] E. Etkina and A. V. Heuvelen, Investigative Science Learning Environment: Using the processes of science and cognitive strategies to learn physics, in Physics Education Research Conference 2001, Rochester, New York, 2001 (2001), http://www.compadre.org/Repository/document/ ServeFile.cfm?ID=4379\&DocID;=1021.

[76] E. F. Redish and D. Hammer, Reinventing college physics for biologists: Explicating an epistemological curriculum, Am. J. Phys. 77, 629 (2009).

[77] A. Madsen, S. B. McKagan, and E. C. Sayre, How physics instruction impacts students' beliefs about learning physics: A meta-analysis of 24 studies, Phys. Rev. ST Phys. Educ. Res. 11, 010115 (2015).

[78] W. G. Perry, Jr., Forms of Intellectual and Ethical Development in the College Years: A Scheme (Holt, Rinehart, and Winston, New York, 1970).

[79] M. F. Belenky, Women's Ways of Knowing: The Development of Self, Voice, and Mind (Basic Books, New York, 1986).

[80] M. Schommer, Effects of beliefs about the nature of knowledge on comprehension, J. Educ. Psychol. 82, 498 (1990).

[81] B. K. Hofer and P. R. Pintrich, The development of epistemological theories: Beliefs about knowledge and knowing and their relation to learning, Rev. Educ. Res. 67, 88 (1997).

[82] K. R. Muis and B. Gierus, Beliefs about knowledge, knowing, and learning: Differences across knowledge types in physics, J. Exp. Educ. 82, 408 (2014).

[83] L. Louca, A. Elby, D. Hammer, and T. Kagey, Epistemological resources: Applying a new epistemological framework to science instruction, Educ. Child Psychol. 39, 57 (2004). 
[84] L. Lising and A. Elby, The impact of epistemology on learning: A case study from introductory physics, Am. J. Phys. 73, 372 (2005).

[85] W. A. Sandoval and K. Morrison, High school students' ideas about theories and theory change after a biological inquiry unit, J. Res. Sci. Teach. 40, 369 (2003).

[86] D. Kienhues, R. Bromme, and E. Stahl, Changing epistemological beliefs: The unexpected impact of a short-term intervention, Br. J. Educ. Psychol. 78, 545 (2008).

[87] T. Porsch and R. Bromme, Effects of epistemological sensitization on source choices, Instr. Sci. 39, 805 (2011).

[88] D. Hammer and A. Elby, On the form of a personal epistemology, Personal Epistemology: The Psychology of Beliefs about Knowledge and Knowing (L. Erlbaum Associates, Mahwah, 2002), pp. 169-190.

[89] D. Hammer and A. Elby, Tapping epistemological resources for learning physics, J. Learn. Sci. 12, 53 (2003).

[90] E. F. Redish, A theoretical framework for physics education research: Modeling student thinking, arXiv: physics/0411149.

[91] G. Bateson, A theory of play and fantasy, in Steps to an Ecology of Mind: Collected Essays in Anthropology, Psychology, Evolution and Epistemology edited by G. Bateson (Ballantine, New York, 1972), pp. 177-193.

[92] E. Goffman, Frame Analysis: An Essay on the Organization of Experience (Harvard University Press, Cambridge, MA, 1974).

[93] D. Tannen, Framing in Discourse (Oxford University Press, New York, 1993).

[94] C. C. van de Sande and J. G. Greeno, Achieving alignment of perspectival framings in problem-solving discourse, J. Learn. Sci. 21, 1 (2012).

[95] K. E. Black and M. C. Wittmann, Epistemic games in integration: Modeling resource choice, AIP Conf. Proc. 951, 53 (2007).

[96] J. Tuminaro and E. F. Redish, Elements of a cognitive model of physics problem solving: Epistemic games, Phys. Rev. ST Phys. Educ. Res. 3, 020101 (2007).

[97] A.H. Schoenfeld, Mathematical Problem Solving (Academic Press, New York, 1985).

[98] C. Baily and N. D. Finkelstein, Interpretation in Quantum Physics as Hidden Curriculum, arXiv:1108.5226.

[99] O. Levrini and P. Fantini, Encountering productive forms of complexity in learning modern physics, Sci. Educ. 22, 1895 (2013).
[100] A. Mason and C. Singh, Do advanced physics students learn from their mistakes without explicit intervention?, Am. J. Phys. 78, 760 (2010).

[101] D. Brookes and E. Etkina, Using conceptual metaphor and functional grammar to explore how language used in physics affects student learning, Phys. Rev. ST Phys. Educ. Res. 3, 010105 (2007).

[102] E. Gire and E. Price, Structural features of algebraic quantum notations, Phys. Rev. ST Phys. Educ. Res. 11, 020109 (2015).

[103] U. Kühne, Die Methode des Gedankenexperiments (2005).

[104] N. J. Nersessian, How do scientists think? capturing the dynamics of conceptual change in science, in Cognitive Models of Science edited by R. N. Giere (University of Minnesota Press, Minneapolis, 1992), p. 3-44.

[105] W. Sandoval, Science education's need for a theory of epistemological development, Sci. Educ. 98, 383 (2014).

[106] C. W. Shubert and D.C. Meredith, Stimulated recall interviews for describing pragmatic epistemology, Phys. Rev. ST Phys. Educ. Res. 11, 020138 (2015).

[107] R. S. Russ, V. R. Lee, and B. L. Sherin, Framing in cognitive clinical interviews about intuitive science knowledge: Dynamic student understandings of the discourse interaction, Sci. Educ. 96, 573 (2012).

[108] K. Charmaz, Constructing Grounded Theory: A Practical Guide through Qualitative Research (Sage Publications Ltd., London, 2006).

[109] E. C. Sayre and P. W. Irving, Brief, embedded, spontaneous metacognitive talk indicates thinking like a physicist, Phys. Rev. ST Phys. Educ. Res. 11, 020121 (2015).

[110] E. Thelen and L. B. Smith, A Dynamic Systems Approach to the Development of Cognition and Action (MIT Press, Cambridge, MA, 2006), Chap. 6, pp. 258-312.

[111] C. Yu and L. B. Smith, Embodied attention and word learning by toddlers, Cognition 125, 244 (2012).

[112] P. N. Kugler and M. T. Turvey, Information, Natural Law, and the Self-assembly of Rhythmic Movement (Routledge, 2015).

[113] J. Minstrell, Explaining the 'at rest' condition of an object, Phys. Teach. 20, 10 (1982).

[114] D. E. Brown and J. Clement, Overcoming misconceptions via analogical reasoning: Abstract transfer versus explanatory model construction, Instr. Sci. 18, 237 (1989). 